\title{
A GEOMETRIC MODEL FOR THE
}

\section{GENERALIZED SYMMETRIC GROUP}

\author{
Norman W. Johnson \\ (received August 18, 1959)
}

The generalized symmetric group $S(n, m)$ consists of all permutations of $\mathrm{mn}$ symbols commutative with

$$
\left(1_{1} 2_{1} \cdot m_{1}\right)\left(1_{2} 2_{2} \ldots m_{2}\right) \cdot\left(1_{n} 2_{n} \cdot \cdot m_{n}\right) .
$$

Since each cycle $Q_{i}=\left(l_{i} 2_{i} \ldots m_{i}\right)$ is of order $m$, there are $\mathrm{m}^{\mathrm{n}}$ permutations within the $\mathrm{n}$ cycles, generating an invariant subgroup 2 of order $\mathrm{m}^{\mathrm{n}}$. Also, there are $\mathrm{n}$ ! ways of permuting the cycles among themselves, by transformations

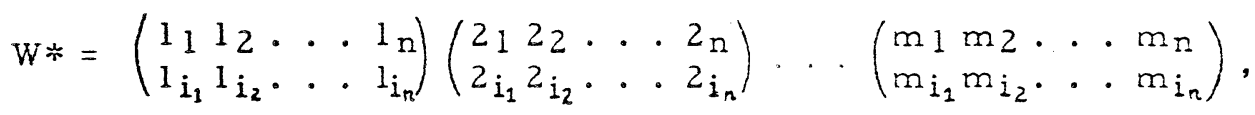

where $i_{1}, i_{2}, \ldots ., i_{n}$ are the symbols $1,2, \ldots, n$ in some order [5, p. 39]. The permutations $W^{*}$ form a subgroup $S_{n} *$ of order $n !$, isomorphic to the symmetric group $S_{n}$.

Clearly $S(n, m)=Q S_{n} *$, and $Q \cap S_{n} *=1$. Hence $S(n, m)$ is of order $m^{n_{n}}$ !, and $S(n, m) / Q \cong S_{n}$. The elements $P$ of $S(n, m)$ can be expressed in the form $P=W * Q$, where $W * \in S_{n} *$ and

$$
Q=Q_{1}{ }^{e_{1}} Q_{2}{ }^{e_{2}} \ldots Q_{n} e_{n}\left(0 \leqslant e_{i} \leqslant m-1\right) .
$$

For $m=1$, the generalized symmetric group reduces to the ordinary symmetric group; i.e., $S(n, 1)=S_{n}$, which is the complete symmetry group of the regular $(n-1)$-dimensional simplex $\alpha_{n-1}$, the analogue of the tetrahedron [3, p. 133]. For $m=2$, the generalized symmetric group becomes the hyper-octahedral group $S(n, 2)$, which is the complete symmetry group of the $n$-dimensional cross polytope $\beta_{n}$, the analogue of the octahedron $[2$, p. $287 ; 3$, p. 133]. Equivalently, $S(n, 2)$

Can. Math. Bull. vol. 3, no. 2, May 1960 
is the complete symmetry group of the n-dimensional measure polytope $\gamma_{n}$, the analogue of the cube, which is reciprocal to $\beta \mathrm{n}[3, \mathrm{p} .133]$. In this paper a geometric interpretation will likewise be given for $S(n, m)$ when $m \geqslant 3$.

Let a segment of length a be denoted by the symbol \{\}$a$. Then the rectangular product \{\}$a \times\{\} b$ is defined to be a rectangle of sides $a$ and $b$. A regular polygon of $m$ sides of length a will be denoted by the symbol $\{\mathrm{m}\} \mathrm{a}$. The square $\{4\} a$ is the rectangular product of two equal segments \{\}a. That is, \{\}$a \times\{\} a=(\{\} a)^{2}=\{4\} a$. A segment of unspecified length will be denoted by \{\} , and a regular $m-g o n$ of unspecified size, by $\{m\}$. Thus one may write \{\}$^{2}=\{4\}$, or, regarding a segment and a square as measure polytopes, $\gamma_{1}{ }^{2}=\gamma_{2}$.

The rectangular product \{\}$a \times\{m\}$ is defined as the right prism of height a whose bases are regular $m-g o n s$ of side $b[1, p .351]$. Its $m$ lateral faces are, of course, rectangles \{\}$a \times\{\} b$. When $a=b$, the lateral faces are squares, and the prism is uniform. If, further, $m=4$, the prism is a cube $\{4,3\} a$. This symbol is to be interpreted as representing a polyhedron with square faces, three of which meet at each vertex, whose edges are of length a. When it is not desired to specify the length of its edges, a cube may be denoted simply by $\{4,3\}$. The general regular polyhedron bounded by $\{p\} a^{\prime} s, q$ at each vertex, has the symbol $\{p, q\}$ a or $\{p, q\} .1)$

This process may be extended indefinitely to yield generalized prisms in higher space. Thus \{\}$a \times\{p, q\} b$, the rectangular product of a segment and a regular polyhedron, is a four-dimensional polytope bounded by two $\{p, q\} b^{\prime} s$, its bases, and $\mathrm{N}_{2}(\mathrm{p}, \mathrm{q})$ lateral cells, each of which is a right prism \{\}$a \times\{p\} b$, where $N_{2}(p, q)$ is the number of faces of a $\{p, q\}$. Such a polytope is called a right polyhedral prism. 2) When $a=b$, the lateral cells are uniform prisms, and the polyhedral prism is uniform. If in addition $\{p, q\}$ is the cube

1) All of these symbols are instances or extensions of a general symbol for regular polytopes devised by the Swiss mathematician Ludwig Schlufli and are accordingly known as Schldfli symbols.

2) Manning [4, p. 237] calls this figure a right hyperprism. 


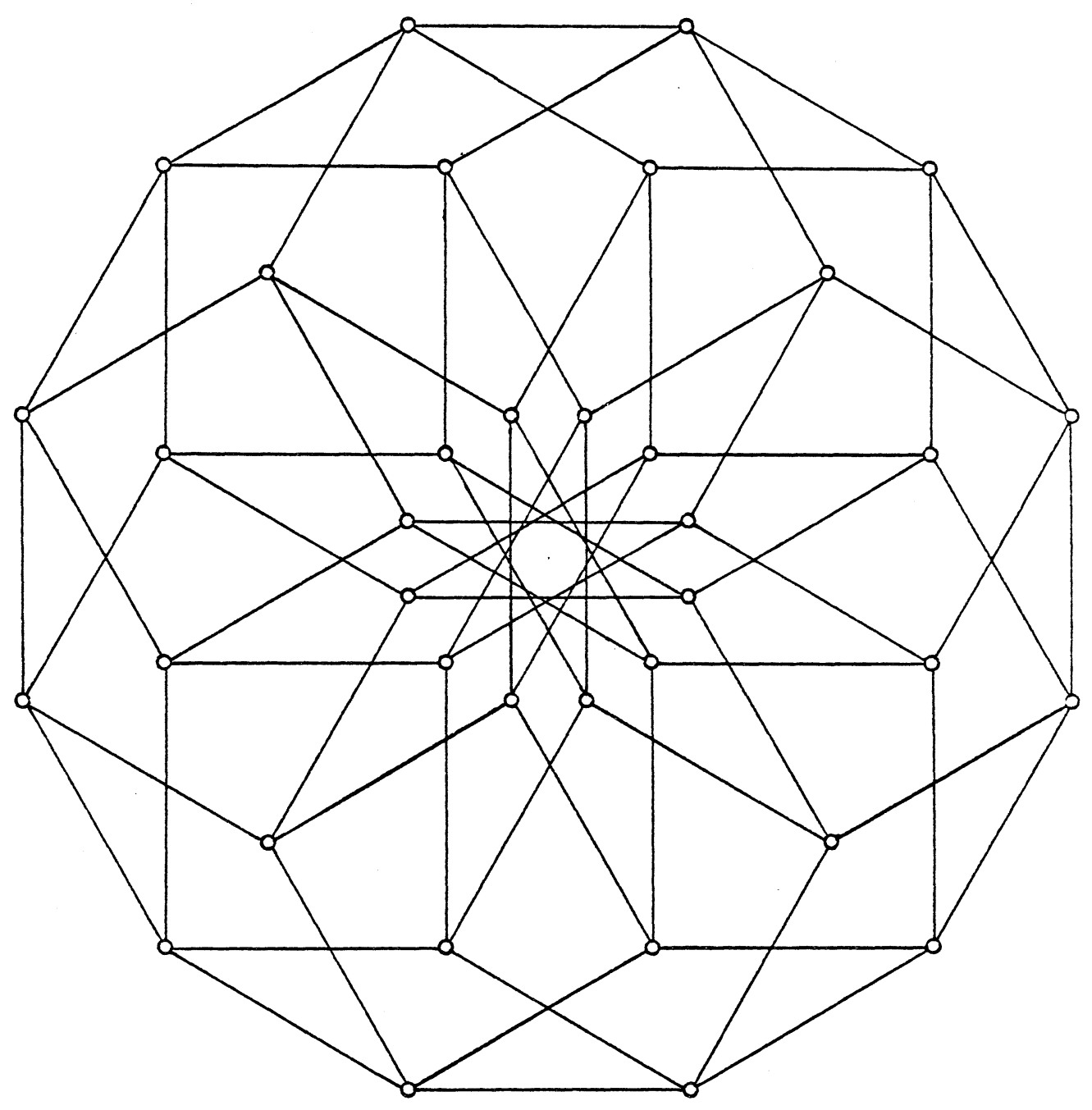

fig. 1 
$\{4,3\}$, the polytope is a tesseract $\{4,3,3\}$ a. This symbol indicates a polytope with cubic cells, three of which meet at each edge, whose edges are of length a.

It is not necessary for the bases of such a four-dimensional prism to be regular. Any polyhedron will do, but if the prism is to be uniform, the bases must be uniform polyhedra, that is to say, polyhedra with regular faces and equivalent vertices, and \{\}a must be of length equal to that of an edge of a base $[1$, p. $351(4.21)]$.

However, this is not the only means of obtaining a fourdimensional prism. Another way is to form the rectangular product of two polygons $\{\mathrm{m}\} \mathrm{a} \times\{\mathrm{p}\} \mathrm{b}[3, \mathrm{p} .124]$. The resulting $m$-gonal $p$-gonal prism ${ }^{3)}$ is bounded by $p$ prisms \{\}$b \times\{m\} a$ and $m$ prisms \{\}$a \times\{p\} b$. When $a=b$, both sets of prisms are uniform, and the polytope is uniform. If, moreover, $m=p$, then all the prisms are alike. A polytope of this kind will be called a polygonal double prism. An $\mathrm{m}$-gonal double prism is denoted by $(\{m\} a)^{2}$ or $\{m\}{ }^{2}$. When $m=4$, this is the tesseract. An orthogonal projection of a hexagonal double prism is shown in fig. 1 .

The general situation is as follows [1, pp. 350-353; 3 , pp. 123-124]. Let $\Pi_{i}, \Pi_{j}, \ldots, \Pi_{s}$ be polytopes of $i, j, \ldots, s$ dimensions. Then the rectangular product $\pi_{i} \times \pi_{j} \times \ldots \times \pi_{s}$ is a prism of $i+j+\ldots+s$ dimensions, with constituents $\pi_{i}, \pi_{j}, \ldots, \pi_{s}$. The order of the factors is immaterial. Furthermore, if we denote the prism $\Pi_{i} \times \Pi_{j}$ by $\Pi_{i+j}$ and the prism $\Pi_{j} \times \Pi_{k}$ by $\Pi_{j+k}$, we have $\Pi_{i} \times \Pi_{j} \times \Pi_{k}=\Pi_{i+j} \times \Pi_{k}=\Pi_{i} \times \Pi_{j+k}$. Thus rectangular-product multiplication is commutative and associative.

A measure polytope $\gamma \mathrm{n}$ can be expressed as the rectangular product of other measure polytopes in $p(n)$ - 1 ways, where $p(n)$ is the number of partitions of $n$. For example,

$$
\gamma_{5}=\gamma_{1} \times \gamma_{4}=\gamma_{2} \times \gamma_{3}=\gamma_{1}^{2} \times \gamma_{3}=\gamma_{1} \times \gamma_{2}^{2}=\gamma_{1}^{3} \times \gamma_{2}=\gamma_{1}^{5} \text {. }
$$

3) Manning [4, p. 248]calls such a figure a right double prism, but I prefer to give the term "double prism" a more restricted meaning. 
A prism whose constituents are all alike will be called a multiple prism. A prism generated by equal polygons is a polygonal multiple prism; one generated by equal polyhedra is a polyhedral multiple prism; etc. A measure polytope $\gamma_{n}$ is a multiple prism in $\tau(n)-1$ ways, where $\tau(n)$ is the number of divisors of n. For example,

$$
\gamma_{6}=\gamma_{3} 2=\gamma_{2}{ }^{3}=\gamma_{1}^{6} \text {. }
$$

Thus, $\gamma_{6}$ may be regarded indifferently as a cubic double prism, a square triple prism, or a right sextuple prism. The multiple prisms of chief importance from the standpoint of this paper are those of the type $\{m\}^{n}$, i.e., regular polygonal multiple prisms. The number of dimensions of such a multiple prism is, of course, $2 \mathrm{n}$.

In four dimensions two planes may be absolutely perpendicular, intersecting only in a point. Since in four dimensions rotation takes place in a plane and about a plane, it is possible to have a rotation simultaneously in each of two absolutely perpendicular planes, leaving only their point of intersection invariant. This combination of rotations through two independent angles is called a double rotation [4, p. 145].

Likewise, in six dimensions rotation is in a plane and about a 4-space. As three mutually perpendicular 4-spaces intersect only in a point, a simultaneous rotation in each of three absolutely perpendicular planes, leaving only their point of intersection fixed, is possible. Such a combination of rotations through three independent angles is called a triple rotation. The general case is that in $2 n$ dimensions one may have a multiple rotation through $\mathrm{n}$ independent angles [3, p. 225].

Consider a prism whose constituents are $\mathrm{n}$ regular polygons, say

$$
\left\{m_{1}\right\} a_{1} \times\left\{m_{2}\right\} a_{2} \times \ldots \times\left\{m_{n}\right\} a_{n},
$$

and assume for the present that no two constituents are alike.4) The faces of this prism consist of $\frac{1}{2} n(n-1) m_{1} m_{2} \cdot \cdot m_{n}$

4) That is, for all $i, j, 1 \leqslant i<j \leqslant n,\left\{m_{i}\right\} a_{i}$ and $\left\{m_{j}\right\} a_{j}$ differ in that either $m_{i} \neq m_{j}$ or $a_{i} \neq a_{j}$ or both. 
rectangles and $m_{1} m_{2} \cdots m_{n} / m_{i} \quad\left\{m_{i}\right\} a_{i}^{\prime} s, 1 \leqslant i \leqslant n$. The faces $\left\{m_{i}\right\} a_{i}$ are parallel to one a nother in much the same way that the $m$ lateral edges of an ordinary prism \{\}$\times\{m\}$, are parallel, and the plane of any face $\left\{m_{i}\right\} a_{i}$ is absolutely perpendicular to the plane of any face $\left\{m_{j}\right\} a_{j}$ for $i \neq j$. Thus the prism may be referred to a $2 n$-dimensional system of rectangular coordinates with the origin at the centre of the prism, so that the faces $\left\{m_{i}\right\} a_{i}$ are parallel to the $x_{2 i-1} x_{2 i}$-plane, which we may call the "i-th plane" [cf. 2, p. 287].

Let $Q_{i}$ be a rotation parallel to the $i$-th plane, about the $(2 n-2)$ - space determined by the remaining $2 n-2$ coordinate axes, through an angle of $2 \pi / \mathrm{m}_{\mathrm{i}}$. This is a symmetry operation of the prism, concurrently taking each vertex of every face $\left\{m_{i}\right\} a_{i}$ into the next one, while leaving fixed all the faces $\left\{m_{j}\right\} a_{j}, j \neq i$. Then $Q_{1}, Q_{2}, \ldots ., Q_{n}$ generate the multiple rotation group $Q \mathrm{~m}_{1} \mathrm{~m}_{2} \ldots \mathrm{m}_{n}$, the direct product of $\mathrm{n}$ cyclic groups:

$$
\mathrm{C}_{\mathrm{m}_{1}} \times \mathrm{C}_{\mathrm{m}_{2}} \times \cdots \times \mathrm{C}_{\mathrm{m}_{n}}
$$

This group is isomorphic to the permutation group represented by

$$
\left(1_{1} 2_{1} \cdots m_{11}\right)^{e_{1}}\left(1_{2} 2_{2} \ldots m_{22}\right)^{e_{2}} \cdot\left(1_{n} 2_{n} \cdot \cdot m_{n n}\right)^{e_{n}}
$$

and is of order $m_{1} m_{2} \ldots m_{n}$.

If some, or all, of the $n$ constituents are alike, there will also be transformations which permute these among themselves. The corresponding isometry is an interchange of the $i$-th and $j$-th planes, which is effected by a half-turn $T_{i j}$ about the $(2 n-2)$ - space

$$
x_{2 i-1}=x_{2 j-1}, \quad x_{2 i}=x_{2 j}
$$

Suppose now that $\mathrm{n}_{1}$ constituents are of one kind, $\mathrm{n}_{2}$ constituents are of a second kind, etc., so that the prism is denoted by

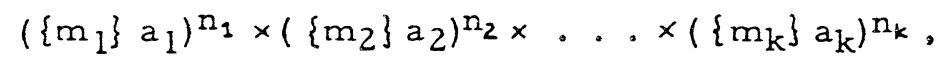

where $\mathrm{n}_{1}+\mathrm{n}_{2}+\ldots+\mathrm{n}_{\mathrm{k}}=\mathrm{n}$. In order to distinguish the identical constituents, let them be further indexed as 


$$
\begin{aligned}
& \left\{m_{11}\right\} a_{11},\left\{m_{12}\right\} a_{12}, \ldots,\left\{m_{1 n_{1}}\right\} a_{1 n_{1}} ; \\
& \left\{m_{21}\right\} a_{21},\left\{m_{22}\right\} a_{22}, \ldots,\left\{m_{2 n_{2}}\right\} a_{2 n_{2}} ; \\
& \cdot ., \cdot, \cdot, \cdot, \cdot, \\
& \left\{m_{k 1}\right\} a_{k 1},\left\{m_{k 2}\right\} a_{k 2}, \ldots,\left\{m_{k n_{k}}\right\} a_{k_{n}} .
\end{aligned}
$$

Similarly re-index the coordinate axes as

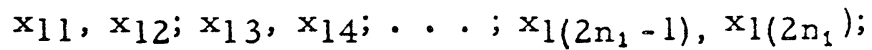

$$
\begin{aligned}
& \mathrm{x}_{21}, \mathrm{x}_{22} ; \mathrm{x}_{23}, \mathrm{x}_{24} ; . . \cdot \mathrm{x}_{2}\left(2 \mathrm{n}_{2}-1\right), \mathrm{x}_{2}\left(2 \mathrm{n}_{2}\right) ; \\
& \mathrm{x}_{\mathrm{k} 1}, \mathrm{x}_{\mathrm{k} 2} ; \mathrm{x}_{\mathrm{k} 3}, \mathrm{x}_{\mathrm{k} 4} ; \ldots ; \mathrm{x}_{\mathrm{k}}\left(2 \mathrm{n}_{\mathrm{k}}-1\right), \mathrm{x}_{\mathrm{k}\left(2 \mathrm{r}_{\mathrm{k}}\right)} \text {; }
\end{aligned}
$$

so that the faces $\left\{m_{h_{i}}\right\} a_{h_{i}}$ are parallel to the $x_{h_{2 i-1}} x_{h_{2 i}}$-plane.

For $1 \leqslant i<j \leqslant n h(1 \leqslant h \leqslant k)$, the half-turn $T_{h_{i j}}$ interchanges the set of faces $\left\{m_{h_{i}}\right\} a_{h_{i}}$ with the set of faces $\left\{m_{h_{j}}\right\} a_{h_{j}}$. The totality of half-turns $T_{l_{i j}}, T_{2_{i j}}, \ldots, T_{k_{i j}}$ generates a group $S_{\mathrm{n}_{1}} \mathrm{n}_{2} \ldots \mathrm{n}_{\mathrm{k}}$ of order $\mathrm{n}_{1} ! \mathrm{n}_{2} ! \ldots \mathrm{n}_{\mathrm{k}} !$, the direct product of $\mathrm{k}$ symmetric groups:

$$
S_{n_{1}} \times S_{n_{2}} \times \cdots \times S_{n_{k}}
$$

It is apparent that $q_{m_{1}} m_{2} \ldots m_{k} \cap S_{n_{1}}^{*} n_{2} \ldots n_{k}=1$. Combining these two groups, we obtain the augmented multiple rotation group.

$$
\mathrm{S}\left(\mathrm{n}_{1}, \mathrm{n}_{2}, \ldots, \mathrm{n}_{\mathrm{k}} ; \mathrm{m}_{1}, \mathrm{~m}_{2}, \ldots, \mathrm{m}_{\mathrm{k}}\right) .
$$

Its order is $m_{1}^{n_{1}} m_{2} n_{2} \ldots . m_{k} n_{k n_{1} ! n_{2} ! \ldots . . n_{k} !}$.

Now suppose that all $n$ constituents are alike. This is the case of the multiple prism $\{m\}^{n}$. Since $k=1$, we now write $m$ for $m_{k}, n$ for $n_{k}, x$ for $x_{k}$, and $T$ for $T_{k}$. The multiple rotation group $2 m_{1} m_{2} \ldots m_{n}$ becomes the group $Q$ mentioned at the beginning of the paper. The group $S_{n} *$ is isomorphic to the group previously defined by the permutations $W *$. Hence the generalized symmetric group $S(n, m)=Q S_{n} *$ can be interpreted, for $m \geqslant 3$, as the augmented multiple rotation group of the polygonal multiple prism $\{m\}^{n}$. 
Osima $[5, p .41]$ defines an element $Q=Q_{1} e_{1} Q_{2} e_{2} \ldots Q_{n} e_{n}$ of $Q\left(0 \leqslant e_{i} \leqslant m-1\right)$ as being of type $\left(n_{0}, n_{1}, \ldots, n_{m-1}\right)$ if the number of $e_{i}$ for which $e_{i}=k$ is $n_{k}$. In terms of our model, a multiple rotation $Q$ is of type $\left(n_{0}, n_{1}, \ldots, n_{m-1}\right)$ if for $n_{k}$ distinct values of $i$ the sets of faces $\{m\}$ parallel to the i-th plane are each rotated through an angle of $2 \mathrm{k} \pi / \mathrm{m}$, the rctation being in each case from the positive half of the $x_{2 i-1-}$ axis towards.the posi-

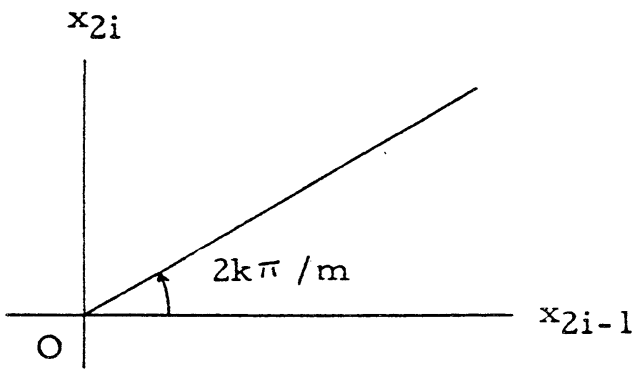
tive half of the $\times 2 i$ axis (see fig. 2).

As previously indicated, the multiple rotation group of $\{m\}^{n}$ can be generated by $n$ rotations $Q_{i}$ of type $(n-1,1,0, \ldots ., 0)$, each of which rotates the faces parallel to the i-th plane through an angle of $2 \pi / \mathrm{m}$ while leaving fixed the faces parallel to the remaining $n-1$ planes. The augmented multiple rotation group is then obtained by combining the multiple rotations with permutations of the $n$ planes of rotation. Thus the representation of $S(n, m)$ as the augmented multiple rotation group of $\{m\}^{n}$ is of degree $n$.

Osima obtains the following formula $[5, \mathrm{p} .44(2.11)]$ for the degree $f_{\alpha^{*}}$ of an irreducible representation of $S(n, m)$ corresponding to the star diagram $[\alpha] \mathrm{m}^{*}=\left[\alpha_{0}\right] \cdot\left[\alpha_{1}\right] \ldots \ldots\left[\alpha_{m-1}\right]$, where $\left[\alpha_{i}\right]$ is an irreducible representation of $S_{n_{i}}$ with degree $f_{\alpha_{i}}$ :

$$
f_{\alpha}{ }^{*}=\frac{n !}{n_{0} ! n_{1} ! \cdot \cdot \cdot n_{m-1} !} f_{\alpha_{0}} f_{\alpha_{1}} \cdot \cdot f_{\alpha_{m-1}} .
$$

In this case, $\mathrm{n}_{0}=\mathrm{n}-1, \mathrm{n}_{1}=1, \mathrm{n}_{2}=\ldots .=\mathrm{n}_{\mathrm{m}-1}=0$, $f_{\alpha_{i}}=1(0 \leqslant i \leqslant m-1)$, so that

$$
f_{\alpha^{*}}=\frac{n !}{(n-1) ! 1 !}=n \text {, }
$$

in agreement with the above. 
A qualification must be added to the foregoing remarks in the event $m=4$. The multiple prism $\{4\} \mathrm{n}$ is identical with the measure polytope $\gamma_{2 n}$. But $\gamma_{2 n}$ can be regarded as a square multiple prism in as many ways as its $2 \mathrm{n}$ constituents $\gamma_{1}$ can be partitioned into $n$ sets of two, namely $(2 n-1)(2 n-3) \cdot . \cdot 1$. Thus for $n \geqslant 2$ the order of the augmented multiple rotation group of $\{4\} n$ is not $4 n_{n}$ ! but $(2 n-1)(2 n-3) . .44^{n} n$. A tesseract, for instance, can be taken as a square double prism in three ways, and its augmented double rotation group is of order $3.4^{2} \cdot 2 !=96$.

To prove that two elements $Q$ and $Q^{\prime}$ of $Q$ are conjugate in $S(n, m)$ if and only if they are of the same type, Osima uses. the representation of $Q_{i}$ as the cyclic group generated by a primitive m-th root of unity. This representation of $Q_{i}$ is obviously isomorphic to its representation as one component of a multiple rotation [cf, also 2, p. 287].

This suggests the possibility of regarding the $x_{2 i-1}$-axis and the $x_{2 i}$-axis $(1 \leqslant i \leqslant n)$ as the respective real and imaginary axes of an Argand plane. That is, the real $2 n$-space of our model can be replaced by unitary $n$-space. The $m^{n}$ vertices of the multiple prism $\{m\}^{n}$ may then be taken as the vertices of a regular complex polytope analogous to the measure polytope $\gamma_{n}$. This generalized measure polytope is denoted by $\gamma \mathrm{m}_{\mathrm{n}}(\mathrm{m}>1, \mathrm{n} \geqslant 1)$. The polytope $\gamma^{2}$ in this notation ${ }^{5} T_{\text {is }}$ the same as the ordinary (real) measure polytope $\gamma_{\mathrm{n}}[6, \mathrm{p} .96 ; 7, \mathrm{p} .374]$.

The complete symmetry group of $\gamma m_{n}$, called $G(m, 1, n)$ by Shephard and Todd [8, pp. 276-277], is readily seen to be isomorphic to $S(n, m)$. The rotations $Q_{i}$ of $\{m\} n$ correspond to unitary reflections (of period $m$ ) of $\gamma m_{n}$, and the half-turns $T_{i j}$ of $\{m\}^{n}$ correspond to 2 -fold reflections of $\gamma^{m} n$. Thus the generalized measure polytope provides an alternative model for the generalized symmetric group.

5) The $m$ in the symbol $\gamma^{m}$ is a superscript, not an exponent. The form $\gamma^{m}$, rather than $\gamma_{n} m$, has been used in order to avoid confusion with the symbol for the rectangular product of $m$ equal $\gamma_{n}{ }^{\prime} s$. 


\section{REFERENCES}

1. H.S.M. Coxeter, The polytopes with regular-prismatic vertex figures, Philos. Trans. Roy. Soc. London Ser. A 229 (1930), 329-425.

2. $\ldots \ldots \ldots \ldots \ldots$, The abstract groups $R^{m}=S^{m}=\left(R^{j} S^{j}\right) P^{j}=1$, $S^{m}=T^{2}=\left(S^{j} T\right)^{2} p_{j}=1$, and $S^{m}=T^{2}=\left(S^{-j} T S^{j} T\right) p_{j}=1$, Proc. London Math. Soc. (2) 41 (1936), 278-301.

3. ........... Regular Polytopes (London, 1948; New York, 1949).

4. H.P. Manning, Geometry of Four Dimensions (New York, 1914).

5. M. Osima, On the representations of the generalized symmetric group, Math. J. Okayama Univ. 4 (1954), 39-56.

6. G.C. Shephard, Regular complex polytopes, Proc. London Math. Soc. (3) 2 (1952), 82-97.

7. ............ Unitary groups generated by reflections, Canad. J. Math. 5 (1953), 364-383.

8. G.C. Shephard and J.A. Todd, Finite unitary reflection groups, Canad. J. Math. 6 (1954), 274-304.

University of Toronto 